\title{
Preliminary inconclusive results of a randomised double blinded cross-over pilot trial in long-term-care dwelling elderly assessing the feasibility of stochastic resonance whole-body vibration
}

\author{
Slavko Rogan ${ }^{1,5 \dagger}$, Lorenz Radlinger ${ }^{1}$, Dietmar Schmidtbleicher ${ }^{2}$, Rob A. de Bie ${ }^{3,4}$ and Eling D. de Bruin ${ }^{3,4,5^{*}+}$
}

\begin{abstract}
Background: This randomised double-blinded controlled cross-over pilot study examined feasibility and preliminary effects of stochastic resonance whole-body vibration training applied in long term care elderly.

Findings: Nine long term care elderly were recruited and randomized to group A (6 Hz, Noise 4 SR-WBV/ Sham) or B (Sham / 1 Hz, Noise 1 SR-WBV). Feasibility outcomes included recruitment rate, attrition, adherence and safety. Physical performance outcomes focused on the Expanded Timed Get Up-and-Go (ETGUG) test, the Short Physical Performance Battery (SPPB), and lower extremity muscle strength.

Of 24 subjects initially approached 9 started and 5 completed the study resulting in 37.5 recruitment, 44.4 attrition and $81.7 \%$ adherence rates. No adverse events were reported. There is more evidence of improved performance levels in the SR-WBV treatment group with significant differences in average change for isometric rate of force development ( $p=0.016$ left leg; $p=0.028$ right leg). No statistical significance was reached for other parameters.

Conclusions: The findings of this study indicate that the used training protocol for long term care elderly is feasible, however, requires more closely monitoring of participants; e.g. needs protocol modifications that target improved compliance with the intervention in this setting. SR-WBV shows beneficial effects on physical performance for those adhering to the intervention.
\end{abstract}

Trial registration: U.S. National Institutes of Health NCT01543243

Physical activity (PA) for elderly is one of the major elements for general health prevention [1] and inactive or sedentary elderly should increase their PA [2]. Despite the known benefits of PA, residents living in long-term care (LTC) are relatively sedentary [3, 4]. Low baseline fitness and mobility levels in (pre-)frail elderly should be considered when starting exercise and this exercise

\footnotetext{
* Correspondence: eling.debruin@hest.ethz.ch

${ }^{\dagger}$ Equal contributors

${ }^{3}$ Department of Epidemiology, CAPHRI School for Public Health and Primary Care, Maastricht University, PO Box 616, 6200 MD, Maastricht, The Netherlands ${ }^{4}$ Centre for Evidence Based Physiotherapy, Maastricht University, PO Box 616, 6200 MD, Maastricht, The Netherlands

Full list of author information is available at the end of the article
}

should be adapted to the physical capabilities of these individuals [5].

Whole body vibration (WBV) seems a safe and beneficial type of balance exercise [6,7]. Pilot studies showed that stochastic resonance WBV (SR-WBV) in (untrained) elderly is both safe and feasible [8, 9]. SR-WBV might also be valuable for (pre-)frail elderly in LTC where the neuromuscular systems of the trainees might not be able withstanding higher loading and long training sessions [8]. However, confirmatory results of such positive effects of WBV in LTC settings is not available and no evidence concerning the feasibility of SR-WBV in LTC dwelling elderly exists. 
This study tested the feasibility and effects of SR-WBV training in LTC elderly with the aim to (I) evaluate the intervention process and the ability to recruit and retain LTC elderly for such an intervention, and (II) assess the impact of 4-week SR-WBV on physical performance.

\section{Findings}

Design

Nine LTC elderly (88.5 \pm 6 years; height: $168 \pm 1 \mathrm{~cm}$; weight: $68.8 \pm 14.3 \mathrm{~kg}$ ) from "Senevita Residenz Multengut, LTC division Muri, Switzerland" volunteered in this randomised double blind controlled cross-over pilot study. Stratified by sex, participants were randomly assigned to group A or B by means of sealed opaque envelopes distributed after baseline assessments. Following Ethical Committee (Canton Berne) approval, informed consent was obtained prior to training (ClinicalTrial.gov: NCT01543243).

Inclusion criteria were aged over 65 years, being able to stand with or without aids, being classified as Resident Assessment Instrument (RAI [10]) performance level $>0$, scoring $>22$ points on the Mini-Mental Status Examination.

\section{Protocol}

Participants were exposed to SR-WBV using a Zeptor med $^{\oplus}$ device (Frei Swiss AG, Zurich, Switzerland). Participants stood freely on both legs wearing comfortable shoes with slight flexion of the hips, knees and ankle joints. In period 1 , participants in group A received 5 sets of $1 \mathrm{~min}$ SR-WBV with $6 \mathrm{~Hz}$, Noise 4 with 1 min of rest between sets, three times a week, during four weeks. One day rest between training sessions was warranted. Group B received a sham intervention of 5 sets of $1 \mathrm{~min}$ SR-WBV with $1 \mathrm{~Hz}$, Noise 1 . The $1 \mathrm{~Hz}$ frequency is expected to cause no training effect [8]. After a wash-out period of 16 days, treatment cross-over took place (Fig. 1). Secondary outcomes were scheduled at baseline (T0) before training, after four weeks training (T1) in period 1, and after four weeks training in period 2 .

\section{Recruitment rate, rate of loss, program adherence}

The criteria for success were based on the primary feasibility objective focussing on recruitment, attrition and adherence to the intervention [11]. Recruitment of a third of the residents deemed eligible, a 15 attrition rate, and $70 \%$ training attendance rate were deemed acceptable [12].

\section{Secondary outcomes}

The reliable Expanded Timed Get Up-and-Go (ETGUG) assessment measured time series of functionally important tasks [13] with details of the protocol reported elsewhere [8]. Physical performance was assessed with the short physical performance battery (SPPB), valid and reliable for lower extremity functions [14] and predictive for disability [15]. A dynamometer tested maximum isometric force (IMVC) and isometric rate of force

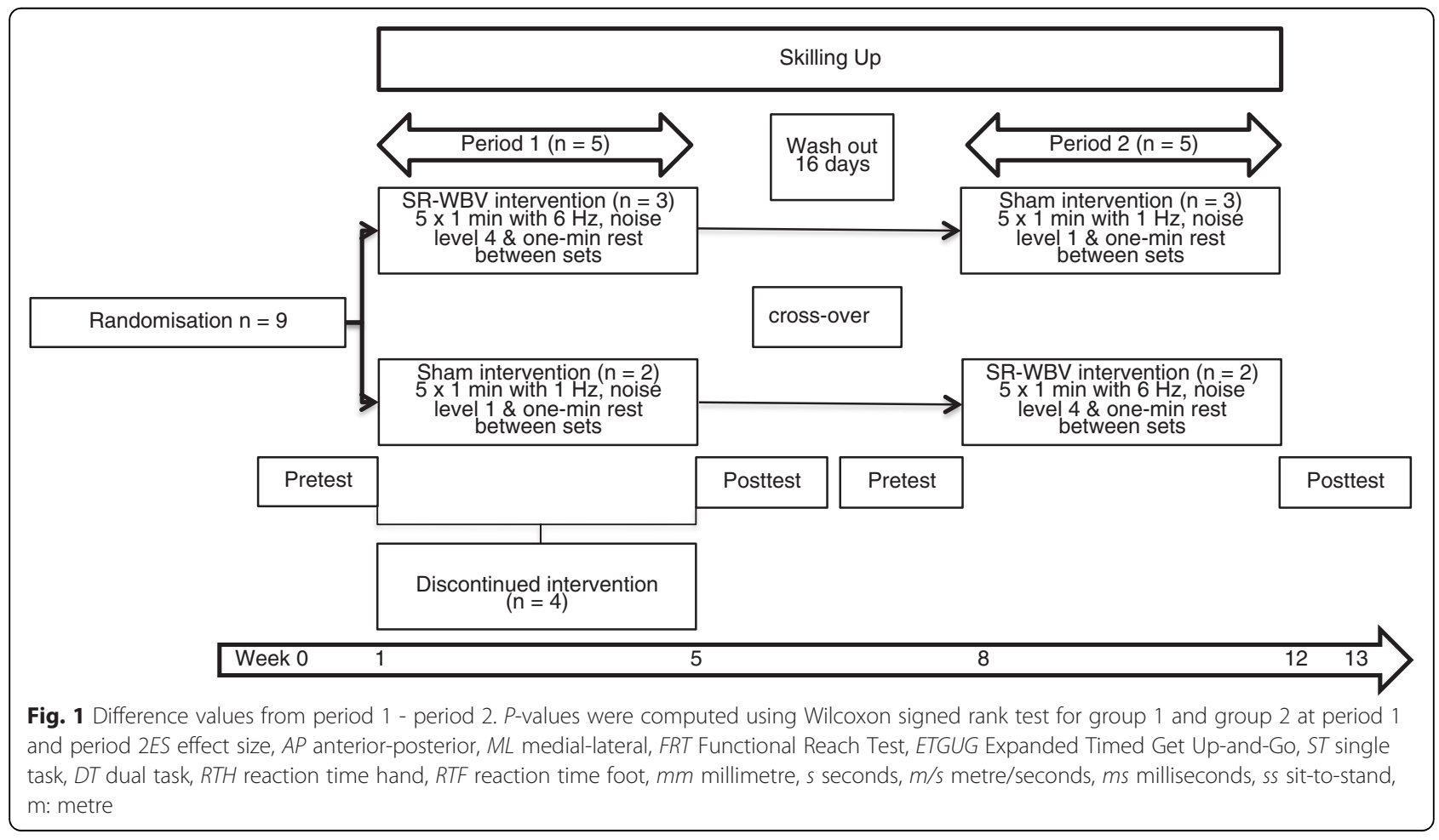


development (IRFD) of the knee extensors (3 trials of testing were averaged to improve reliability [16]). IRFD was determined from the force-time curve calculating the steepness between the time points of 25 and $75 \%$ IMVC. Details of the protocol were previously published [16].

\section{Statistical analysis}

For recruitment, data for the total sampling frame for inclusion in the trial were taken. For the inclusion rate-i.e. the proportion of participants invited to participate who enrolled into the study-we distinguished between those who refused, did not respond or who were willing but excluded. Compliance to the treatment protocol was calculated using "Number of vibration sessions $\div$ the total number of possible vibration sessions $\times 100$ ". Nonparametric Rank-Order Tests of Puri and Sen L Statistics [17] assessed change over time. Effect sizes (ES) were calculated for the differences within and between the groups and expressed as $r=Z / \sqrt{ } \mathrm{N}$; where $r 0.1$ is considered a "small", 0.3 a "medium" and $\geq 0.5$, a 'large' effect [18].

\section{Recruitment, attrition, and adherence}

Tweenty four residents were deemed eligible to participate based on the inclusion-exclusion criteria and invited to participate. Nine LTC elderly individuals consented to partake resulting in $37.5 \%$ recruitment rate. Fifteen participants declined with reasons given: no interest $(n=8)$, no motivation $(n=6)$ and personal reasons $(n=1)$. Five participants finished both study periods (age: $85.3 \pm 6$ years; height: $161.4 \pm 6.2 \mathrm{~cm}$; weight: $72.9 \pm 15.9 \mathrm{~kg}$ ) (Additional file 1) resulting in an attrition rate of $44.4 \%(n=4$ dropouts). The reasons for dropping out were: not motivated to continue $(n=3)$, and heart disease $(n=1)$. Adherence to the intervention for those continuing with training resulted in $81.7 \%$ (98 of 120 training sessions) compliance.

\section{Secondary outcomes}

Table 1 presents the test results and Table 2 summarises the intervention effects with ES per group for both periods. There are no carry-over effects for any of the outcome measures (Table 1), however, within group effect size for the SPPB $(p=0.039)$ is large. Between group effects with large ES are seen for the ETGUG 0 to $2 \mathrm{~m}$, ETGUG 2 to $8 \mathrm{~m}$, IRFD left and IRFD right.

\section{Discussing the findings}

The present pilot study aimed to develop and test a SRWBV exercise intervention in LTC. The main focus of this study was to evaluate the feasibility of the SR-WBV intervention and the ability to recruit and retain LTC elderly, and to assess the effects of the intervention. Both the aging population and the number of institutionalized older people are expected to increase in the future. Clinical research in long term care is, however, rather still
Table 1 Repeated measures analyses of variance for ranked data for cross-over effects

\begin{tabular}{llll}
\hline & Pillai's trace $\left(r^{2}=S S_{\text {Bet }} / S S_{\text {Tot }}\right)$ & $L\left[(N-1) r^{2}\right]$ & Probability \\
\hline ETGUG SS & 0.208 & 0.789 & 0.440 \\
ETGUG 0-2 m & 0.536 & 3.462 & 0.160 \\
ETGUG 2-8 m & 0.931 & 4,467 & 0.332 \\
ETGUG turn & 0.833 & 5.000 & 0.167 \\
ETGUG 12-18 m & 0.833 & 5.000 & 0.167 \\
ETGUG 18-20 m & 0.556 & 1,250 & 0.444 \\
ETGUG total time & 0.833 & 5.000 & 0.167 \\
SPPB & 0.932 & 4.559 & 0.329 \\
IMVC, left & 0.556 & 3.750 & 0.148 \\
IMVC, right & 0.556 & 1.250 & 0.444 \\
IRFD, left & 0.772 & 2.600 & 0.278 \\
IRFD, right & 0.893 & 2.778 & 0.409 \\
\hline ETGUG Expanded Timed
\end{tabular}

ETGUG Expanded Timed Get Up-and-Go, IMVC isometric maximal voluntary contraction, IRFD isometric rate of force development, SPPB Short Physical Performance Battery, ss sit to stand movement

scarce. Research is, therefore, essential to improve the quality of care in LTC homes. Quality of care and improvement thereof in LTC for the aged is, amongst others, relient on future evidence from research projects and their feasibility in real life conditions.

We demonstrated the feasibility of acquiring acceptable recruitment and compliance rates for LTC dwelling older people randomised in this clinical trial. Our targets of 33 recruitment and $70 \%$ compliance of those training were attained; e.g. those remaining in the study showed excellent compliance with the exercise intervention and retesting. Compared with median rates for recruitment and adherence in falls prevention interventions in institutional settings for clinical trials [12] we achieved better or similar rates for these measures. However, our trial suffered from a rather high attrition rate which we mainly attribute to motivational aspects, e.g. seventeen individuals reported to be not interested or motivated to either begin with exercising or continue to do so. Illness and personal reasons for withdrawal were only explaining training discontinuation of two individuals. Five of initially nine individuals completed the training programme and retest data were obtained from these individuals. Thus, our trial protocoll in its current form is deemed not feasible in LTC because of the high attrition rate. A future study should aim for an attrition rate of around $15 \%$ [12].

From previous studies we know that the presence of a professional exercise instructor working in the facility was significantly associated with exercise participation and with higher exercise frequencies and levels, and session duration [4]. Furthermore, programs must be carefully designed and coached in order to prevent attrition [19] and be focused on the motivation performing functional activities in the LTC setting [20]. Fairhall et al. 
Table 2 Results for physical performance within and between SR-WBV and Sham intervention

\begin{tabular}{|c|c|c|c|c|c|}
\hline & \multirow{2}{*}{$\begin{array}{l}\text { SR-WBV }(n=5) \\
\text { Median }(\mathrm{IQ})\end{array}$} & \multirow{2}{*}{$\begin{array}{l}p \text { Within intervention } \\
\text { ES }\end{array}$} & \multirow{2}{*}{$\begin{array}{l}\text { Sham }(n=5) \\
\text { Median }(\mathrm{IQ})\end{array}$} & \multirow{2}{*}{$\begin{array}{l}p \text { Within intervention } \\
\text { ES }\end{array}$} & \multirow{2}{*}{$\begin{array}{l}p \text { Between intervention } \\
\text { ES }\end{array}$} \\
\hline & & & & & \\
\hline \multicolumn{6}{|l|}{ SPPB } \\
\hline pretest & $5.0(3-5.5)$ & $0.039^{*}$ & $6.0(4.5-7)$ & 0.10 & 0.396 \\
\hline posttest & $6.0(4.5-8)$ & 0.92 & $6.0(2.5-6.5)$ & 0.73 & 0.27 \\
\hline \multicolumn{6}{|c|}{ ETGUG sS, (s) } \\
\hline pretest & $1.9(1.2-2.7)$ & 0.080 & $1.5(0.9-2.0)$ & 0.080 & 0.421 \\
\hline posttest & $1.6(0.9-2.0)$ & 0.79 & $1.8(1.3-3.3)$ & 0.78 & 0.30 \\
\hline \multicolumn{6}{|c|}{ ETGUG 0-2 m (s) } \\
\hline pretest & $3.0(2.4-3.9)$ & 0.225 & $2.5(2.1-2.9)$ & 0.138 & 0.117 \\
\hline posttest & $2.4(2.1-3.0)$ & 0.54 & $3.2(2.3-3.9)$ & 0.67 & 0.50 \\
\hline \multicolumn{6}{|c|}{ ETGUG 2-8 m, (s) } \\
\hline pretest & $8.3(6.8-12.0)$ & $0.043^{*}$ & $7.4(6.0-8.0)$ & $0.043^{*}$ & $0.009^{*}$ \\
\hline posttest & $7.0(6.0-7.8)$ & 0.91 & $8.8(8.2-9.9)$ & 0.91 & 0.83 \\
\hline \multicolumn{6}{|c|}{ ETGUG turn, (s) } \\
\hline pretest & $9.5(8.2-12.9)$ & $0.043^{*}$ & $8.4(6.9-9.3)$ & $0.042^{*}$ & 0.402 \\
\hline posttest & $9.0(6.9-9.4)$ & 0.91 & $9.1(7.7-10.0)$ & 0.91 & 0.26 \\
\hline \multicolumn{6}{|c|}{ ETGUG 12-18 m, (s) } \\
\hline pretest & $8.3(6.6-12.4)$ & $0.043^{*}$ & $7.4(6.0-9.0)$ & $0.043^{*}$ & 0.347 \\
\hline posttest & $8.1(6.0-9.3)$ & 0.91 & $8.2(7.9-11.0)$ & 0.91 & 0.30 \\
\hline \multicolumn{6}{|c|}{ ETGUG 18-20 m, (s) } \\
\hline pretest & $6.8(5.9-9.7)$ & 0.345 & $6.5(5.5-8.4)$ & $0.025^{*}$ & 0.917 \\
\hline posttest & $6.8(5.5-8.4)$ & 0.42 & $6.8(6.3-8.4)$ & 0.54 & 0.03 \\
\hline \multicolumn{6}{|c|}{ ETGUG total time, (s) } \\
\hline pretest & $38.9(30.8-52.6)$ & $0.043^{*}$ & $34.1(28.3-38.5)$ & $0.043^{*}$ & 0.076 \\
\hline posttest & $35.2(28.3-28.9)$ & 0.91 & $37.8(35.8-44.1)$ & 0.91 & 0.56 \\
\hline \multicolumn{6}{|c|}{ IMVC left, N } \\
\hline pretest & $293(63-698)$ & 0.138 & $210(137-522)$ & 0.345 & 0.251 \\
\hline posttest & $210(136-793)$ & 0.66 & $166(31-1032)$ & 0.42 & 0.36 \\
\hline \multicolumn{6}{|c|}{ IMVC right, N } \\
\hline pretest & $241(67.4-595)$ & $0.043^{*}$ & $241(130-711)$ & 0.80 & 0.465 \\
\hline posttest & $282(75-752)$ & 0.91 & $184(63-521)$ & 0.79 & 0.23 \\
\hline \multicolumn{6}{|c|}{ IRFD left, N/s } \\
\hline pretest & $504(185-1131)$ & $0.043^{*}$ & $417(130-3074)$ & 0.080 & $0.016^{*}$ \\
\hline posttest & $937(631-2120)$ & 0.91 & $67(35-384)$ & 0.79 & 0.76 \\
\hline \multicolumn{6}{|c|}{ IRFD right, N/s } \\
\hline pretest & $325(138-816)$ & $0.043^{*}$ & $417(130-3074)$ & 0.068 & $0.028^{*}$ \\
\hline posttest & $862(617-2405)$ & 0.91 & $212(112-505)$ & 0.82 & 0.68 \\
\hline
\end{tabular}

Median and interquartile range (IQR) values are at baseline (pre) and after intervention (post). P-values after pretest and posttest intervention were computed using the Wilcoxon signed ranks test within intervention and Mann-Whitney $U$ tests for SR-WBV intervention and sham-intervention SBBP Short Physical performance Battery, ETGUG Expanded Timed Get Up-and-Go, ss sit to stand movement, IMVC isometric maximum voluntary contraction, IRFD isometric rate of force development, ES effect size, s seconds, $\mathrm{N} / \mathrm{s}$ Newton/seconds

*Statistically significant difference $(p<0.05)$ after pre and post intervention 
[21] postulated that frail elderly individuals should be encouraged and supported to adhere to an intervention plan and studies show higher compliance and fewer dropouts for exercise when the program is accompanied by Motivation-Volition (MoVo) programs [22].

Although we are aware of the fact that the emphasis of a pilot study should be placed on feasibility and not on statistical significance [11] our data allow creating a sample size table for various values of the effect or variance estimates to acknowledge the uncertainty surrounding the pilot estimates. For example, based on an estimated meaningful change in SPPB score of 1 point [23], a significance level set at $5 \%$, a power of $80 \%$ to detect differences with two-sided hypothesis testing, inclusion of $N=30$ participants ( $n=15$ per group) will be needed for a future two-groups pre- post-test study design. It should be stressed, however, that this sample size calculation should be interpreted with caution because our estimates may be unrealistic or biased because of the limited sample size [11].

This pilot study provided useful information about the feasibility of the experimental intervention that used SRWBV for training. Our subjects tolerated the weekly physical intervention well. Those compliant to training were able to progress in intensity and duration of the exercises. Furthermore, our experience suggests that our SR-WBV was of sufficient duration and/or intensity to ameliorate muscle capacity as indicated by improvements in the secondary outcomes. Pragmatically, however, our experimental subjects did not keep up their motivation to perform regular training three times a week, nor were they willing to do so. We believe, therefore, that it is necessary to proceed to an additional study in LTC, however, with major modifications to the protocol. The modifications should thereby focus on improving the motivation to train in LTC dwelling individuals and also assess benefits or risks [24] of this type of training for older people.

Summarising the findings and limitations of this study it becomes clear that this study only reveals first estimates for the chosen outcome measures. We implemented a strict study design to control threats to validity. A necessary next step would be to adapt the study protocol by adding an intervention component that emphasises motivational aspects of exercising and, thus, strive to improve attrition rates in a new LTC SR-WBV exercise group study design as an additional control procedure.

\section{Conclusions}

We conclude that pilot studies with explicit feasibility objectives and success criteria are important foundation steps in preparing for large trials [11] and for development of rehabilitation research programs. Ongoing formal review of the multifaceted issues inherent in the design and conduct of pilot studies can provide invaluable feasibility and scientific data for rehabilitation specialists working in LTC, e.g. physiotherapists, willing to perform clinical trials [25], and may also be highly relevant for furthering the development of theory based rehabilitation [26]. SR-WBV training as applied in this study is deemed only conditionally feasible; it requires some major modifications to the protocol. However, SRWBV shows trends to stronger improvement in lower extremity muscle properties when compared with sham training. This study encourages the further development of this intervention, preferably with a randomized control design. Future programs must be carefully designed and coached by professional exercise instructors in order to prevent attrition.

\section{Availability of supporting data}

The data set(s) supporting the results of this article are included within the article (and its additional file(s)).

\section{Additional file}

Additional file 1: Description of the demografic variables of the participants included in the analysis. (DOC $31 \mathrm{~kb}$ )

\section{Abbrevations}

ES: Effect size; ETGUG: Expanded Timed Get Up-and-Go; IMVC: Isometric voluntary contraction IRFD, Isometric rate of force development; MMSE: MiniMental Status Examination; MoVo: Motivation-Volition program; PA: Physical activity; SR-WBV: Stochastic resonance whole-body vibration; WBV: Wholebody vibration.

\section{Competing interests}

The authors declare no potential conflict of interest with respect to the authorship and/or publication of this article.

\section{Authors' contributions}

$S R, L R$ and $E d B$ conceived the idea of this study. SR, LR, DS and EdB participated in the conception and design of the study. SR supervised the WBV trainings session and Data collection. SR conducted the statistical analyses. SR and EdB wrote the manuscript. SR, LR, DS, RdB, and EdB were involved in drafting or revising the manuscript critically for its content. All authors read and approved the final manuscript.

\section{Acknowledgement}

We would like to thank Ross Bennie for proof reading.

\section{Author details}

${ }^{1}$ Department Health, Bern University of Applied Sciences, Bern, Switzerland. Department of Sport Science, Wolfgang-Goethe University Frankfurt, Frankfurt, Germany. ${ }^{3}$ Department of Epidemiology, CAPHRI School for Public Health and Primary Care, Maastricht University, PO Box 616, 6200 MD, Maastricht, The Netherlands. ${ }^{4}$ Centre for Evidence Based Physiotherapy, Maastricht University, PO Box 616, 6200 MD, Maastricht, The Netherlands. ${ }^{5}$ Department of Health Sciences and Technology, Institute of Human Movement Sciences and Sport, ETH Zurich, Wolfgang-Pauli-Str. 27, HIT J 31.2, $\mathrm{CH}-8093$, Zurich, Switzerland.

Received: 4 May 2015 Accepted: 18 September 2015

Published online: 09 October 2015 


\section{References}

1. Baert V, Gorus E, Mets T, Geerts C, Bautmans I. Motivators and barriers for physical activity in the oldest old: a systematic review. Ageing Res Rev. 2011:10(4):464-74

2. Freiberger E, Sieber C, Pfeifer K. Physical activity, exercise, and sarcopenia - future challenges. Wien Med Wochenschr. 2011;161(17-18):416-25.

3. Benjamin K, Edwards N, Ploeg J, Legault F. Barriers to physical activity and restorative care for residents in long-term care: a review of the literature. J Aging Phys Act. 2014;22(1):154-65.

4. De Souto BP, Demougeot L, Vellas B, Rolland Y. How much exercise are older adults living in nursing homes doing in daily life? A cross-sectional study. J Sports Sci. 2015;33(2):116-24.

5. Gardner MM, Buchner DM, Robertson MC, Campbell AJ. Practical implementation of an exercise-based falls prevention programme. Age Ageing. 2001;30(1):77-83.

6. Chanou K, Gerodimos V, Karatrantou K, Jamurtas A. Whole-body vibration and rehabilitation of chronic diseases: a review of the literature. J Sports Sci Med. 2012;11(2):187-200

7. Rogan S, Hilfiker R, Herren K, Radlinger L, de Bruin ED. Effects of whole-body vibration on postural control in elderly: a systematic review and meta-analysis. BMC Geriatr. 2011;11:72

8. Rogan S, Radlinger L, Hilfiker R, Schmidtbleicher D, de Bie RA, de Bruin ED. Feasibility and effects of applying stochastic resonance whole-body vibration on untrained elderly: a randomized crossover pilot study. BMC Geriatr. 2015;15:25.

9. Rogan S, Radlinger L, Schmid S, Herren K, Hilfiker R, de Bruin ED. Skilling up for training: a feasibility study investigating acute effects of stochastic resonance whole-body vibration on postural control of older adults. Ageing Res. 2012;1:e5-3.

10. Carpenter I, Hirdes JP. Using interRAl assessment systems to measure and maintain quality of long-term care. In: A Good Life in Old Age? Monitoring and Improving Quality in Long-term Care. OECD Publishing; 2013: 263

11. Thabane L, Ma J, Chu R, Cheng J, Ismaila A, Rios LP, et al. A tutorial on pilot studies: the what, why and how. BMC Med Res Methodol. 2010;10:1

12. Nyman SR, Victor CR. Older people's recruitment, sustained participation, and adherence to falls prevention interventions in institutional settings: a supplement to the Cochrane systematic review. Age Ageing. 2011;40(4):430-6.

13. Botolfsen $P$, Helbostad JL, Moe-Nilssen R, Wall JC. Reliability and concurrent validity of the expanded timed Up-and-Go test in older people with impaired mobility. Physiother Res Int. 2008;13(2):94-106.

14. Guralnik JM, Simonsick EM, Ferrucci L, Glynn RJ, Berkman LF, Blazer DG, et al. A short physical performance battery assessing lower-extremity function - association with self-reported disability and prediction of mortality and nursing-home admission. J Gerontol. 1994;49(2):M85-94.

15. Guralnik JM, Ferrucci L, Simonsick EM, Salive ME, Wallace RB. Lower-extremity function in persons over the Age of 70 years as a predictor of subsequent disability. New Engl J Med. 1995;332(9):556-61.

16. Mebes C, Amstutz A, Luder G, Ziswiler HR, Stettler M, Villiger PM, et al. Isometric rate of force development, maximum voluntary contraction, and balance in women with and without joint hypermobility. Arthritis Rheum. 2008;59(11):1665-9.

17. Thomas JR, Nelson JK, Thomas KT. A generalized rank-order method for nonparametric analysis of data from exercise science: a tutorial. Res Q Exerc Sport. 1999;70(1):11-23.

18. Corder GW, Foreman DI. Nonparameztric statistics for non-statisticans. a step-by-step approach. Hoboken, New Jersey: John Wiley \& Sons. Inc.; 2009.

19. Zech A, Drey M, Freiberger E, Hentschke C, Bauer JM, Sieber CC, et al. Residual effects of muscle strength and muscle power training and detraining on physical function in community-dwelling prefrail older adults: a randomized controlled trial. BMC Geriatr. 2012;12:68.

20. Resnick B. Functional performance of older adults in a long-term care setting. Clin Nurs Res. 1998;7(3):230-46. discussion 246-239.

21. Fairhall N, Langron C, Sherrington C, Lord SR, Kurrle SE, Lockwood K, et al. Treating frailty-a practical guide. BMC Med. 2011;9:83.

22. Fuchs R, Goehner W, Seelig H. Long-term effects of a psychological group intervention on physical exercise and health: the MoVo concept. J Phys Act Health. 2011;8(6):794-803.

23. Kwon S, Perera S, Pahor M, Katula JA, King AC, Groessl EJ, et al. What is a meaningful change in physical performance? Findings from a clinical trial in older adults (the LIFE-P study). J Nutr Health Aging. 2009;13(6):538-44.

24. Brooke-Wavell K, Mansfield NJ. Risks and benefits of whole body vibration training in older people. Age Ageing. 2009;38(3):254-5.
25. Cook DJ, Rocker G, Meade M, Guyatt G, Geerts W, Anderson D, et al. Prophylaxis of Thromboembolism in Critical Care (PROTECT) Trial: a pilot study. J Crit Care. 2005;20(4):364-72.

26. de Bruin ED, van Het Reve E, Murer K. A randomized controlled pilot study assessing the feasibility of combined motor-cognitive training and its effect on gait characteristics in the elderly. Clin Rehabil. 2013;27(3):215-25.

\section{Submit your next manuscript to BioMed Central and take full advantage of:}

- Convenient online submission

- Thorough peer review

- No space constraints or color figure charges

- Immediate publication on acceptance

- Inclusion in PubMed, CAS, Scopus and Google Scholar

- Research which is freely available for redistribution 